\title{
Tracking Solar Events through Iterative Refinement
}

\author{
Dustin J. Kempton ${ }^{\mathrm{a}, *}$, Rafal A. Angryk ${ }^{\mathrm{a}}$ \\ ${ }^{a}$ Department of Computer Science, Georgia State University, P.O. Box 5060, Atlanta, GA 30302-5060, USA
}

\begin{abstract}
In this paper, we combine two approaches to multiple-target tracking: the first is a hierarchical approach to iteratively growing track fragments across gaps in detections; the second is a network flow based optimization method for data association. The network flow based optimization method is utilized for data association in an iteratively growing manner. This process is applied to solar data, retrieved from the Heliophysics Event Knowledge base (HEK) and utilizes precomputed image parameter values. These precomputed image parameter values are used to compare visual similarity of detected events, to determine the best matching track fragment associations, which leads to a globally optimal track fragment association hypothesis.
\end{abstract}

Keywords: Tracking, Sensor Fusion, Iterative Method

\section{Introduction}

With the launch of NASA's Solar Dynamic Observatory (SDO) mission, solar physics researches entered the big data era. SDO's space telescope registers approximately 70,000 high resolution (4096 by 4096) images daily; obtaining one image every ten seconds. Instruments on the SDO space telescope record data at a high spatial resolution and time cadence, which equates to about 0.55 petabytes of raster data per year. This trend in data acquisition in solar physics is expected to grow by even larger volumes with the introduction of the ground-based Daniel K. Inouye Solar Telescope (DKIST) Martens et al. (2012). These large datasets generate a pioneering opportunity to observe correlations between various solar phenomena (events) that have previously gone unexplored.

To facilitate the important needs of space weather monitoring (which can have vital impacts on space and air travel, power grids, GPS, and communication devices), many software modules developed by the Feature Finding Team (FFT), work continuously on the massive SDO raster data, generating object data with spatiotemporal characteristics. The object data with spatiotemporal characteristics generated by FFT software modules is directly reported to the Heliophysics Event Knowledge base (HEK) Hurlburt et al. (2012), and is available to the public online and are searchable through the iSolSearch graphical interface ${ }^{1}$. This spatiotemporal data is used to accurately catalog, explore, track, and correlate solar events.

To accurately capture correlations between solar events, it is essential to develop tracking techniques that are ca-

\footnotetext{
* Corresponding author

Email addresses: dkempton1@cs.gsu.edu (Dustin J. Kempton), angryk@cs.gsu.edu (Rafal A. Angryk)

${ }^{1}$ https://www.lmsal.com/isolsearch
}

pable of handling multiple solar event instance hypotheses concurrently. This concurrent hypothesis requirement arises from the interactions of multiple solar events, in the field of view, simultaneously. The modeling of multiple objects moving through a given space, for the purposes of tracking their movements, is Multiple Hypothesis Tracking, which will be discussed in Section 2 .

This paper expands upon our previous work that was first introduced in Kempton et al. (2014). In that previous work, some of the implementation details were omitted for brevity. This work shall include the indexing techniques utilized to achieve the run times reported previously; specifically, the spatial fixed grid that was utilized to index individual event detections prior to their linking into track fragments is presented in Section 4.5. A modified version of the same fixed grid is also used in indexing track fragments after their initial linking process. An additional indexing structure is presented in this work that was not part of our previous work in Kempton et al. (2014). This structure was added for speeding up the calculation of the expected change in the number of detections from one time frame to the next, and is discussed further in Section 4.4 .

The remainder of this paper is organized by first introducing the concept of Multiple Hypothesis Tracking in Section 2 and its formulation as a maximum a posteriori problem. We then discuss using a track graph to solve the maximum a posteriori problem as the optimal global data association in Section 3. Then we discuss the calculation of the costs of each edge of the track graph in Section 4 along with some other implementation details. We then conclude with some experimental results of the changes made for this work and compare them to our previous results achieved in Kempton et al. (2014). 


\section{Multiple Hypothesis Tracking}

The goal of multiple hypothesis tracking is to estimate the state of one or more moving object. We apply this state modeling technique in the attempt to estimate the state of evolving areas of solar phenomena reported to the HEK as sets of detections from the FFT software modules. In this, it is generally the case that sensor reports do not contain identity information of the targets being reported on as detections. It is also generally understood that some of the reports from the sensors may indeed be false returns, or that the probability of detection is less than one, as no computer vision system has been shown to be correct at all times. Due to the last two observations, it is incorrect to assume that sensor reports in the HEK can be considered as true target observations, they must be considered only as possible target observations. Thus, the most crucial component of any algorithms used for the tracking of multiple targets is the association of sensor reports in the creation of possible target tracks Chong et al. (2009).

\subsection{Hypothesis Formulation as Maximum a Posteriori}

The formulation of hypotheses in this work is done similar to that seen in Zhang et al. (2008) and Hofmann et al. (2013), by defining the association problem as a maximum a posteriori problem. This is done by first letting $O=\left\{o_{i}\right\}$ be a set of detection reports taken from the HEK. Each of the detection responses $o_{i}=\left(x_{i}, s_{i}, a_{i}, t_{i}\right)$ contain information about the object detection, where $x_{i}$ is the spatial position, $s_{i}$ is the spatial representation of the object detection, $a_{i}$ is the physical appearance (e.g., color, or image parameter histogram), and $t_{i}$ is the time stamp of the start of the object detection. A single trajectory hypothesis of one particular object is further defined as an ordered list of object observations, $T_{k}=\left\{o_{k_{1}}, o_{k_{2}}, \ldots, o_{k_{n}}\right\}$ where $o_{k_{i}} \in O$. A global association hypothesis is then defined as a set of single trajectory hypotheses, $\tau=\left\{T_{k}\right\}$ Zhang et al. (2008).

The data association problem is then defined as maximizing the posteriori probability of $\tau$ given the set of observations $O$ :

$$
\begin{aligned}
\tau^{*} & =\underset{\tau}{\operatorname{argmax}} P(\tau \mid O) \\
& =\underset{\tau}{\operatorname{argmax}} P(O \mid \tau) P(\tau) \\
& =\underset{\tau}{\operatorname{argmax}} \prod_{i} P\left(o_{i} \mid \tau\right) P(\tau)
\end{aligned}
$$

For the final iteration of equation 11 to hold, we must assume that the likelihood probabilities are conditionally independent given the hypothesis $\tau$, meaning that knowledge about the truth of hypothesis $\tau$ does not change the belief in the likelihood of seeing observation $o_{i}$. To see that knowledge about the truth of hypothesis $\tau$ does not change the belief in the likelihood of seeing observation $o_{i}$, let us consider the likelihood function of observation for $o_{i}, P\left(o_{i} \mid \tau\right)$ in the next section.

\subsubsection{Conditional Independence of Likelihood}

The likelihood function of observation on $o_{i}$, denoted as $P\left(o_{i} \mid \tau\right)$, is used to model cases of an observation being a true detection verses the observation being a false detection. There are differing ways of modeling this behavior. For instance, Zhang et al. (2008) utilizes a Bernoulli distribution and simply has a constant probability $\beta_{i}$ of $o_{i}$ being a false detection. In Hofmann et al. (2013), a learned relation, between the size $s_{i}$ of $o_{i}$ and the probability of $o_{i}$ being a false or true positive, is derived from annotated data.

It is our belief, that the constant value assumption for $\beta_{i}$ is too simplistic, as not all detections are of equal validity, and their validity is dependent upon the frequency of detections in the recent history. Similarly, we do not have data that is annotated with identity information for use in developing a learned relationship between object properties and their probability of being either a false or true positive detection, as done in Hofmann et al. (2013). This lead us to choose to model the value as a Poisson distribution based upon the count history of recent detections.

The choice of this distribution is based upon the fact that the observation being a false detection is something that can be counted, as is generally expected for this type of distribution. Secondly, occurrences of an observation being a false detection are independent, meaning the fact that a false detection is reported neither diminishes nor increases the chance of another being reported. Third, it is possible to count the number of false detections that are reported but it is meaningless to try and count the number of false detections that have not been reported. The final condition that is generally used to see if a Poisson distribution applies, is the average frequency of an occurrence for a time frame is known.

Our modeling of the likelihood of observation utilizes the average absolute change in the number of detections reported per frame, for the previous $n$ frames. By using the previous $n$ frames, instead of a constant average value, we allow for our model of false detections to vary as periods of more or less dynamic activity occur in the data. To facilitate the efficient calculation of this moving average, a temporal index based upon time frames is utilized and is described in Section 4.4 . Our previous work in Kempton et al. (2014) utilized a static value that was calculated over all frames, whereas the introduction of the temporal index allows for a dynamic value calculated over the previous $n$ frames.

The calculation of the Poisson probability of a detection from a given frame being a false detection is calculated as $f(\Delta ; \lambda)=\operatorname{Pr}(X=\Delta)=\frac{\lambda^{\Delta} e^{-\lambda}}{\Delta !}$, where $\Delta$ is the absolute change in the number of detections from the previous frame to the frame of $o_{i}$ and $\lambda$ is the expected change, which is calculated from the previous $n$ frames. The likelihood of observation $P\left(o_{i} \mid \tau\right)$ is then simply the inverse or $P\left(o_{i} \mid \tau\right)=1-f(\Delta ; \lambda)$ which is labeled as $\beta_{i}$ and is utilized in the calculation of the cost of observation, described later 


\section{in Section 3.3}

As can be seen in the formulation of the likelihood of observation function $P\left(o_{i} \mid \tau\right)$, the knowledge of the truth of track hypothesis $\tau$ does not factor into the calculation and thus does not change the belief in the likelihood of seeing observation $o_{i}$. Thus our original assumption in Equation 1. that the likelihood probabilities are conditionally independent given the hypothesis $\tau$, is correct.

\subsubsection{Association Hypothesis Prior Decomposition}

With the likelihood function of observation defined, we now turn to the prior probability of association hypothesis $\tau$ from Equation 1, labeled as $P(\tau)$. It should be noted, that the optimization of Equation 1 is difficult, due to the combinatorial size of the set of association hypotheses in $\tau$. This requires a reduction in the size of the set of association hypotheses, which is possible with the assumption that one object can belong to one and only one trajectory, i.e. $\tau_{k} \cap \tau_{l}=\emptyset, \forall k \neq l$. Furthermore, by assuming that the motion of each object is independent, Equation 1 can be rewritten as:

$$
\tau^{*}=\underset{\tau}{\operatorname{argmax}} \prod_{i} P\left(o_{i} \mid \tau\right) \prod_{\tau_{k} \in \tau} P\left(\tau_{k}\right)
$$$$
\text { Where } \tau_{k} \cap \tau_{l}=\emptyset, \forall k \neq l
$$

Note that the prior of all trajectories has been broken down to the product of the priors of each trajectory $\prod_{\tau_{k} \in \tau} P\left(\tau_{k}\right)$. In Equation 2, each prior $P\left(\tau_{k}\right)$ is modeled as a Markov chain, which includes an initialization probability $P_{\text {entr }}$ at the detection of its initial time step, a termination probability $P_{\text {exit }}$ at the detection of its final time step $n$, and the transition probabilities $P_{\text {link }}\left(o_{k_{i+1}} \mid o_{k_{i}}\right)$ between each detection in the interval between initial and final time steps. The model is expanded as follows:

$$
\begin{aligned}
P\left(\tau_{k}\right) & =P\left(\left\{o_{k_{o}}, o_{k_{1}}, \ldots, o_{k_{n}}\right\}\right) \\
& =P_{\text {entr }}\left(o_{k_{0}}\right) P_{l i n k}\left(o_{k_{1}} \mid o_{k_{0}}\right) P_{l i n k}\left(o_{k_{2}} \mid o_{k_{1}}\right) \\
& \ldots P_{\text {link }}\left(o_{k_{n}} \mid o_{k_{n-1}}\right) P_{\text {exit }}\left(o_{k_{n}}\right)
\end{aligned}
$$

The exact representations of the entrance probability $P_{\text {entr }}\left(o_{k_{0}}\right)$, exit probability $P_{\text {exit }}\left(o_{k_{n}}\right)$, and transition probability $P_{l i n k}\left(o_{k_{n}} \mid o_{k_{n-1}}\right)$ will be discussed in Section 4.1. In the following section, we turn the discussion to modeling the data association problem as a directed acyclic graph, which is then used to solve the maximum a posteriori problem.

\section{Data Association Through Track Graphs}

The problem of data association can be represented as a directed acyclic graph; in doing so, we convert the maximization of the a posteriori probability problem, presented in Equation 1, to finding the minimum cost multicommodity flow through a graph. We call this graph the

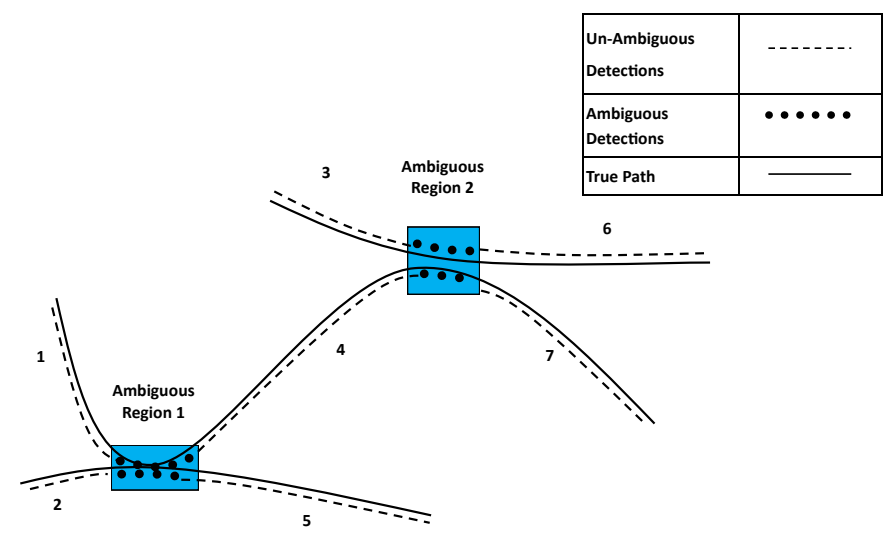

Figure 1: Sensor reports are associated into pure track fragments and ambiguous regions are solved later.

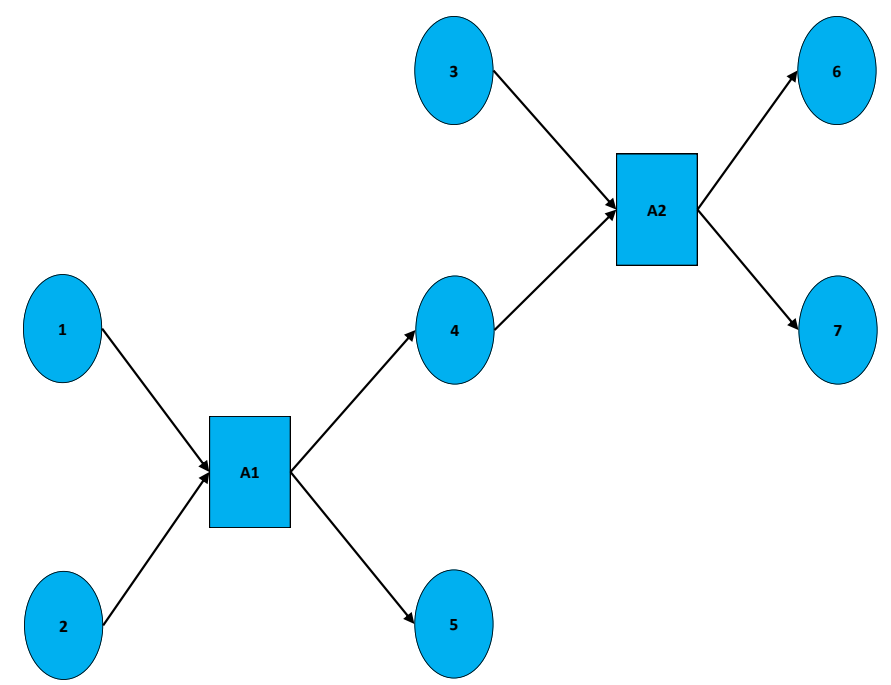

Figure 2: Track fragment graph represents possible associations. Ambiguous regions A1 and A2 would be represented as multiple nodes, as there are multiple detections inside these regions

track fragment graph, and in this graph, the nodes are sensor reports; the reports may be individual detections of an object, or track fragments, that contain multiple detections. For example, in Fig. 1 multiple sensor reports are grouped into track fragments and labeled as nodes 1 through 7 in Fig. 2, when there are no conflicting track paths in the neighborhood. This grouping of detections into fragments is described in more detail in Section 3.2 . Similarly, each of the detections in the ambiguous regions in Fig. 1 will be entered into the track graph as a node individually, as opposed to being grouped together.

Edges/links that connect two nodes are created if and only if the nodes have the possibility of representing the same object trajectory at differing timestamps. To be considered as a possible representation of the same object trajectory, the start time of the successor track fragment must be larger than the end time of the predecessor track fragment.

In the track graph, a hypothesis of an object's move- 
ment is a path in the graph, and a global association hypothesis is a set of consistent tracks. In order to meet the property of being consistent, no two tracks in the global association hypothesis can share a single object detection report $o_{i}$.

\subsection{Managing Hypotheses}

If it is assumed that any object detection report $o_{k_{i}}$ ( $k_{i}$ denotes the $i^{\text {th }}$ frame and the $k^{t h}$ object report in said frame), can be associated with any object detection report in a subsequent frame $o_{k_{i+1}}$, then for $m$ frames with $n$ object reports per frame, the number of all possible track paths is $\theta\left(n^{m}\right)$. This means that the optimal hypothesis is found by solving an NP hard optimization, which grows exponentially in size with the number of report frames Chong (2012).

The graphical formulation of the track association problem combines the reduction power of using track fragments instead of individual reports, and implicitly, rather than explicitly, represents hypotheses as paths through the graph. This approach will still have an exponential rate of growth in the number of track hypotheses, but the number of nodes and edges in the graph grows at a linear rate with respect to the number of tracks in the data. It is because of this linear rate of growth, that the graphical representation was chosen for our work, over an explicit construction of every possible track hypothesis. Similarly, the choice to conservatively group object detections, that have a high probability of being the same tracked object, into track fragments, was also chosen as a computation and memory consumption reduction method.

\subsection{Generation of Track Fragments}

In the generation of a track fragment graph, individual detection reports are first grouped into track fragments. This is done by taking advantage of the the discrete spatiotemporal location of each detection. In other words, each detection report $o_{i}$ is processed independently of a vast majority of the other detection reports in the dataset.

In the processing of a single detection report, only a very limited subset of the dataset can be considered as candidates for the same object detection at the next time step. Specifically, in the conservative grouping into track fragments step, only those detection reports in the time step immediately following the time step of the detection being processed are considered. Furthermore, only those detection reports that also intersect the spatial neighborhood of the processed detection report can be considered. This strict constraint, of using spatiotemporal neighbors, coupled with the constraint, that only those detection reports with one and only one spatiotemporal neighbor can be linked into the same track fragment at this juncture, allows certain data dependency issues to be ignored. This arises because of the fact that when and if two detection reports get added to different track fragments, generated by two different threads of execution, these track fragments

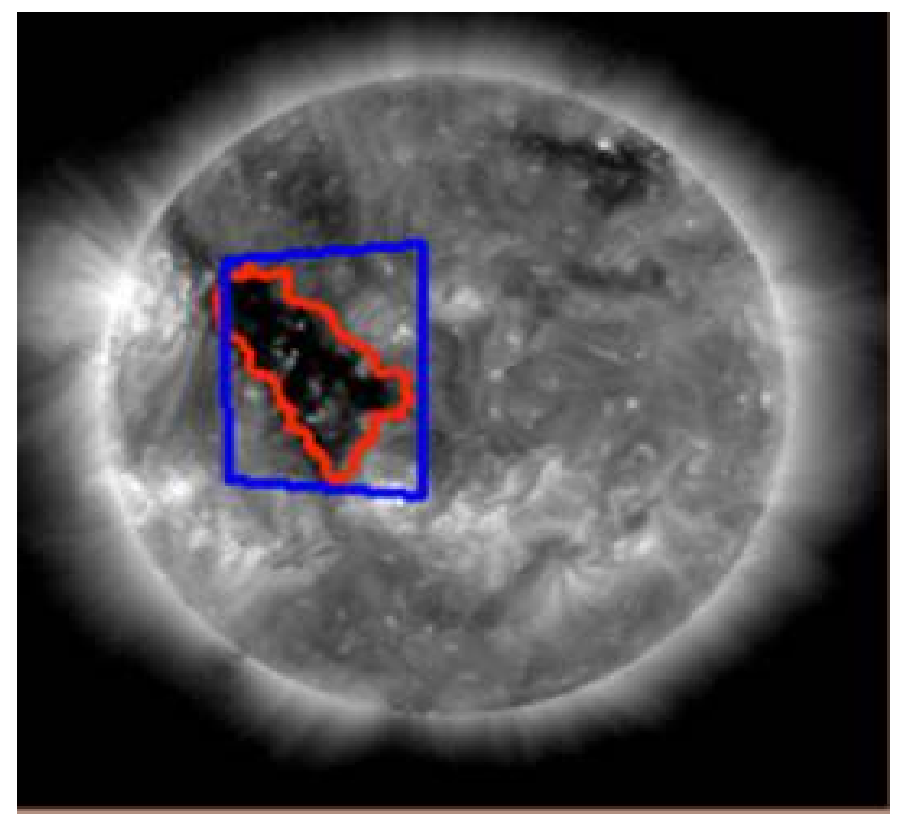

Figure 3: A object detection report (polygon represented as chain code) and search area for the next report (quadrilateral) in subsequent frame.

will be equivalent, by the constraint that only one spatiotemporal neighbor was available for each detection to be linked with. Therefore, any duplicate results produced by parallel execution, can simply be pruned and discarded at the conclusion of the grouping process.

It is this equivalency of duplicate results that allows for a judicious relaxation, or ignoring of, some data dependencies, and this allows for the exploitation of aggressive parallelism as shown in Meng et al. (2010). In that work, it was shown that applications where each iteration is likely to update only a small portion of the model, are highly likely to update different parts of the model, and can tolerate "errors" introduced by executing iterations in parallel, can scale well in parallel processing environments. Similarly, in track fragment generation, "error" in the form of duplicate track fragments, can be tolerated, as the duplicate results can be pruned as was previously stated. Furthermore, numerically equivalent results do not need to come from the parallel portion of an algorithm that fulfills these constraints, as is the case with track fragment generation.

So, the algorithm for track fragment generation can be trivially implemented on a parallel random-access machine. In the generation of track fragments in Algorithm1, each detection report is assumed to be a node in a doublylinked list, with its predecessor and successor initialized to be a null pointer. It is also assumed that the detection reports contain timestamps indicating the start and end of the detection report, which are used to predict the location of the detection report at the beginning of the next time step. Furthermore, it is also assumed that the detection report contains information about the location 


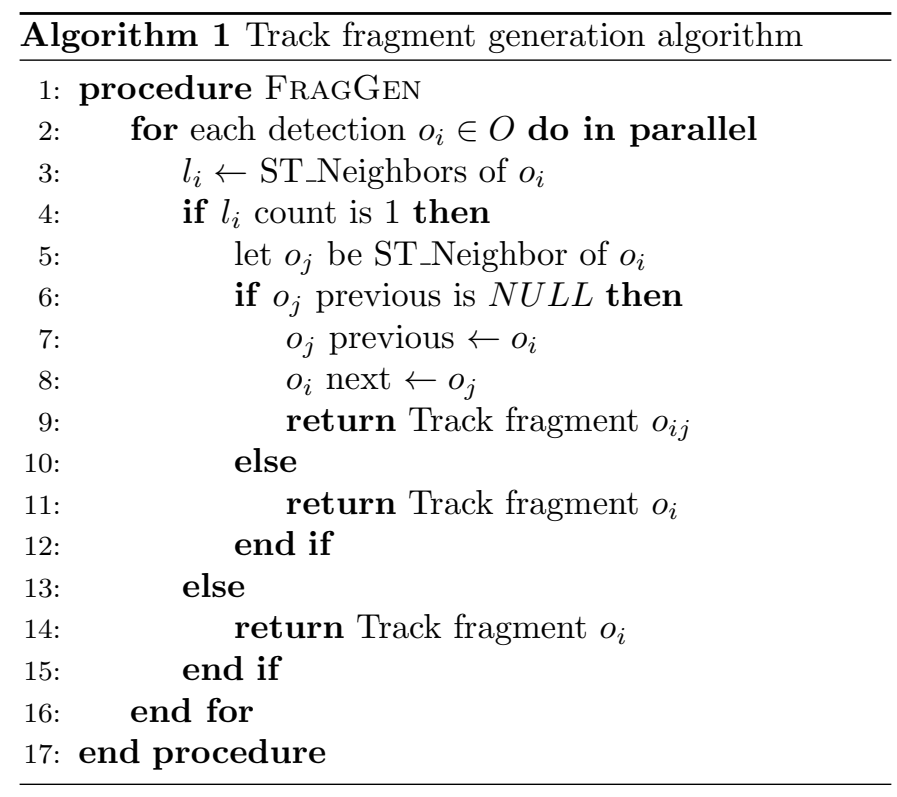

$x_{i}$, and shape of the detection $s_{i}$, which are used to calculate overlap between predicted location, as shown in Fig. 3. and the location of the detection being considered as a candidate for track fragment generation.

As seen in Algorithm 1, each detection report searches for a set of spatiotemporal neighbors in a search area depicted in Fig. 3, and produces a track fragment based upon the count of neighbors, in conjunction with the current state of the node pointers of the found neighbors. This process can produce several duplicate pointers to the same logical track as depicted in Fig. 4. This "error", introduced by the parallel processing, is removed by pruning the duplicate track fragments based upon the end nodes in the doubly linked list. It is this pruning process by list traversal, and the checking to see if a node is already pointed to by another in Algorithm 1, that dictates the use of a doubly-linked list, as opposed to a singly-linked list.

Once the pruning step is complete, the duplicate track fragment pointers are removed from the returned results and only one track fragment pointer remains, as depicted in Fig. 5. The results after Algorithm 1 are not guaranteed to have more than one detection in any particular linked list, though there shall be at least one detection in each linked list. In either case the results are treated similarly in subsequent steps of the tracking process.

\subsection{Track Fragment Graph}

References to the track fragment graph have been relatively informal to this point. In this section, the track graph creation will be formalized as a minimum cost multicommodity flow problem. To begin, first let the following $0-1$ indicator variables of hypothesis membership be defined as in Chong (2012); Zhang et al. (2008); Pirsiavash

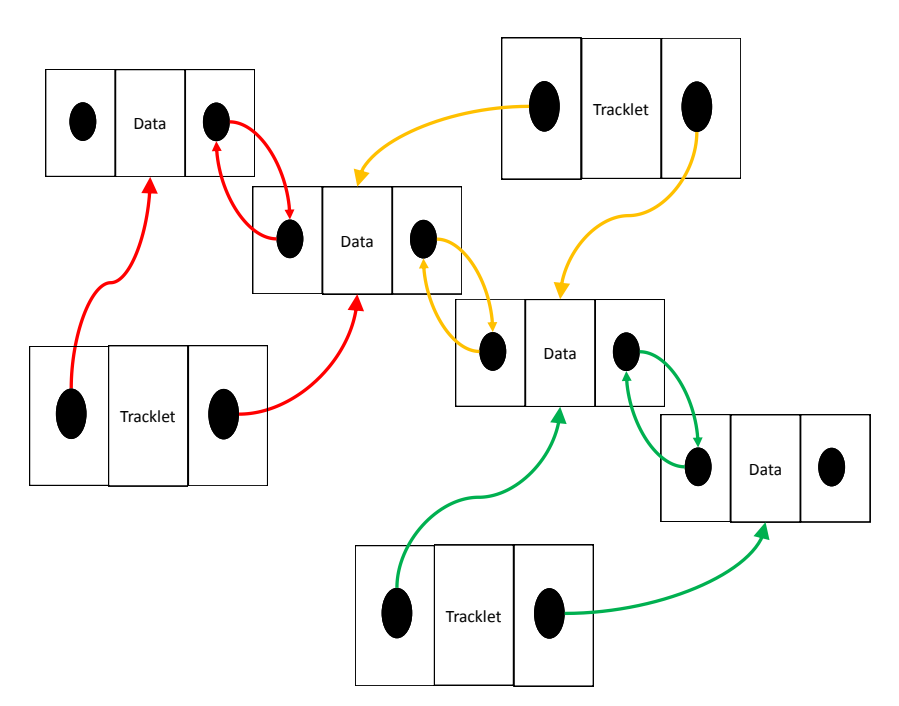

Figure 4: Duplicate track fragments depict the same logical track fragment.

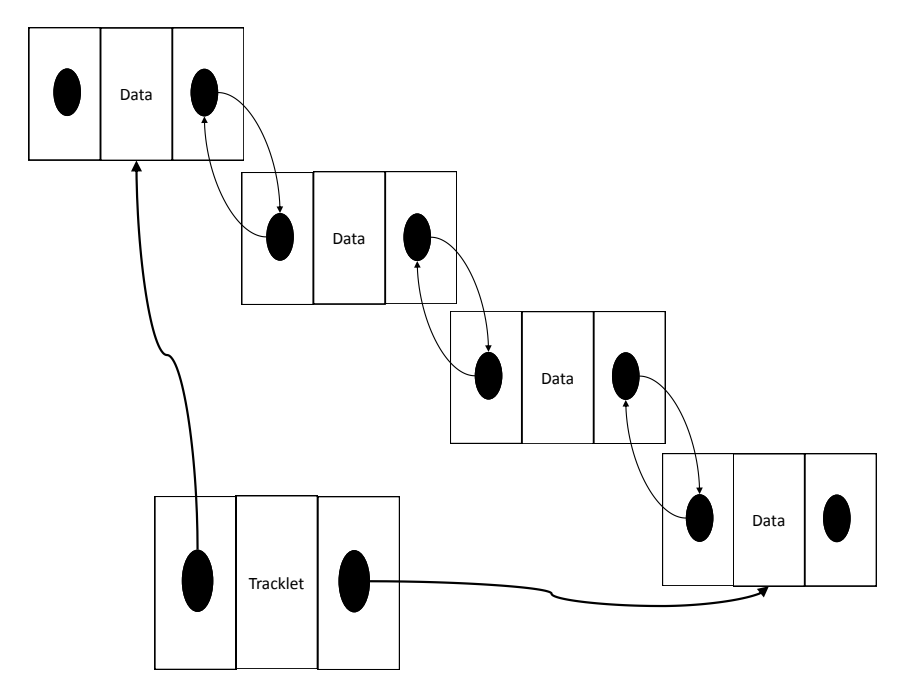

Figure 5: Duplicate track fragments are removed post parallel processing. 
et al. (2011):

$$
\begin{aligned}
f_{\text {en }, i} & = \begin{cases}1 & \exists \tau_{k} \in \tau, \tau_{k} \text { starts from } o_{i} \\
0 & \text { otherwise }\end{cases} \\
f_{\text {ex }, i} & = \begin{cases}1 & \exists \tau_{k} \in \tau, \tau_{k} \text { ends at } o_{i} \\
0 & \text { otherwise }\end{cases} \\
f_{i, j} & = \begin{cases}1 & \exists \tau_{k} \in \tau, o_{j} \text { immediately follows } o_{i} \text { in } \tau_{k} \\
0 & \text { otherwise }\end{cases} \\
f_{i} & = \begin{cases}1 & \exists \tau_{k} \in \tau, o_{i} \\
0 & \text { otherwise }\end{cases}
\end{aligned}
$$

In this case, $o_{i}$ is now considered a track fragment in trajectory hypothesis $\tau_{k}$, as opposed to a single detection report, as was the case in 1. Recall that there is a constraint that track hypotheses are non-overlapping in order for the set to be consistent, and this constraint is met when:

$$
f_{e n, i}+\sum_{j} f_{j, i}=f_{i}=f_{e x, i}+\sum_{j} f_{i, j}
$$

In other words, when the flow into a node in the graph is the same as the flow out of the node, and both are the same as the flow through that same node, that can be attributed to one trajectory hypothesis $\tau_{k}$. In Eq. 5 and 4a. $f_{e n, i}$ is the flow into the node from the source node, which means the beginning of the track is at the referenced node. Similarly, $f_{e x, i}$ of Eq. 5 and $4 \mathrm{~b}$ is the flow out of the node to the termination node, which indicates the end of the track is at the referenced node. The indicator $f_{i, j}$ of Eq. 5 and $4 \mathrm{c}$ is the flow from node $i$ to node $j$ within a given hypothesis. Finally, $f_{i}$ of Eq. 5 and $4 \mathrm{~d}$ indicates the referenced node belongs to the hypothesis of interest $\tau_{k}$.

The conversion of the maximization problem indicated in Eq. 1 into the minimum cost multi-commodity flow problem, the negative log of the posterior is taken as follows:

$$
\begin{aligned}
& \tau^{*}= \underset{\tau}{\operatorname{argmin}} \sum_{i}-\log P\left(o_{i} \mid \tau\right)+\sum_{\tau_{k} \in \tau}-\log P\left(\tau_{k}\right) \\
&=\underset{\tau}{\operatorname{argmin}} \sum_{i}\left(-\log \left(1-\beta_{i}\right) f_{i}-\log \beta_{i}\left(1-f_{i}\right)\right) \\
&+\sum_{\tau_{k} \in \tau}\left(C_{e n, k_{0}} f_{e n, k_{0}}\right. \\
&\left.+\sum_{j}\left(C_{k_{j}, k_{j+1}} f_{k_{j}, k_{j+1}}\right)+C_{e x, k_{n}} f_{e x, k_{n}}\right) \\
&= \underset{\tau}{\operatorname{argmin}} \sum_{i}\left(C_{e n, i} f_{e n, i}\right)+\sum_{i j}\left(C_{i, j} f_{i, j}\right) \\
&+\sum_{i}\left(C_{e x, i} f_{e x, i}\right)+\sum_{i}\left(C_{i} f_{i}\right)
\end{aligned}
$$

The final iteration of Eq. 6 is subject to the flow constraints set out in Eq. 5, and the cost of flow through the nodes are given by the functions defined as follows:

$$
\begin{aligned}
C_{e n, i} & =-\log P_{\text {entr }}\left(o_{i}\right) \\
C_{e x, i} & =-\log P_{\text {exit }}\left(o_{i}\right) \\
C_{i, j} & =-\log P_{\text {link }}\left(o_{j} \mid o_{i}\right)
\end{aligned}
$$

Each of the Markov chain parameters $P_{\text {entr }}\left(o_{i}\right), P_{\text {exit }}\left(o_{i}\right)$, $P_{\text {link }}\left(o_{j} \mid o_{i}\right)$ will be discussed in Section 4.1. Also recall that $\beta_{i}=1-f(\Delta ; \lambda)$ models the cases of an observation being a true detection or a false detection, as seen in Section 2.1 .1

$$
C_{i}=-\log \frac{\beta_{i}}{1-\beta_{i}}
$$

By following the formulation in Eq. 6, utilizing the cost functions Eq. 7a through Eq. 7d, and the hypothesis membership indicators Eq. 4a through Eq. 4d, the cost-flow network $G(X)$ is constructed. The network is initialized with a source node $S$ and a sink node $T$. Using the set of track fragments $O$, that were returned from the track fragment formulation process Algorithm 1, and then pruned for duplicate removal, two nodes $u_{i}$ and $v_{i}$ are created for every track fragment $o_{i} \in O$. An edge $\left(u_{i}, v_{i}\right)$ and an associated cost to that edge of $c\left(u_{i}, v_{i}\right)=C_{i}$, as indicated in Eq. 7d, is also created for each of these node pairs, where $C_{i}$ is considered the cost of observation, or how probable it is that the detection is a false detection. A flow of $f\left(u_{i}, v_{i}\right)=f_{i}$ will also be assigned to this edge where $f_{i}$ is from the indicator variable defined in Eq. $7 \mathrm{~d}$ and is 1 if the track fragment $o_{i}$ is in the hypothesis, and 0 otherwise. The hypothesis membership will be assigned when solving the multi-commodity flow problem later described in Section 3.4.

With the node pairs $u_{i}$ and $v_{i}$ in the graph for every track fragment $o_{i} \in O$, an edge from the source node $S$ is inserted into the graph to every node $u_{i}$. These edges will have an associated cost of $c\left(S, u_{i}\right)=C_{e n, i}$ as indicated in Eq. 7a. which is the cost associated with a track hypothesis beginning at that track fragment. The flow through these edges will be that of the indicator variable in Eq. 4 a as $f\left(S, v_{i}\right)=f_{\text {en, } i}$. Recall that this variable is 1 when the track hypothesis begins at track fragment $o_{i}$ and 0 otherwise.

Similar to the edges inserted from the source node $S$ to all of the nodes $u_{i}$ in the graph, each of the nodes $v_{i}$ will receive an edge going to the terminating node $T$. These edges will have an associated cost of $c\left(v_{i}, T\right)=C_{e x, i}$ as indicated in Eq. $7 \mathrm{~b}$, which is the cost associated with a track hypothesis ending at that track fragment. The flow through these edges will be that of the indicator variable in Eq. $4 \mathrm{~b}$ as $f\left(v_{i}, T\right)=f_{\text {ex }, i}$. Recall that this variable is 1 when the track hypothesis ends at track fragment $o_{i}$ and 0 otherwise.

Once the modeling of hypotheses entering, exiting, and being false detections at particular track fragments for 


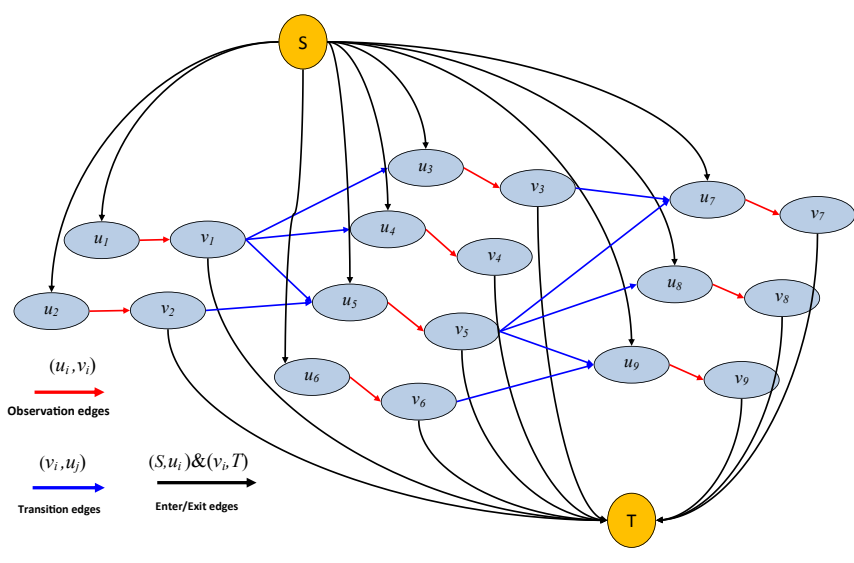

Figure 6: An example of the cost-flow network with three time steps and 9 observations.

$o_{i} \in O$ are entered into $G(X)$, the modeling of possible associations between track fragments must be added to the graph $G(X)$ as well. This is only done for those track fragments that have an association probability of a nonzero value, or $P_{\text {link }} \neq 0$. This is determined by a similar projection of a search area as was done previously in the track fragment generation algorithm, Algorithm 1. Those track fragments determined to be within the search area are given an edge from the track fragment $o_{i}$ to each of the candidates $o_{j}$. The edges are created from node $v_{i}$ of searching track fragment $o_{i}$ to node $u_{j}$ of neighboring fragment $o_{j}$, with an associated cost of $c\left(v_{i}, u_{j}\right)=C_{i, j}$ as noted in Eq. 7c. The flow through the edges are that of the indicator variable in Eq. 4c, where the flow is 1 when the track hypothesis traverses from track fragment $o_{i}$ to fragment $o_{j}$, and will be 0 otherwise. These formulations are presented in Zhang et al. (2008) and are also used in Pirsiavash et al. (2011); Chong (2012). A depiction of what a graph based upon this formulation would look like is shown in Fig. 6

\subsection{Association by Solving the Track Fragment Graph}

With the track fragment graph $G(X)$ constructed, the data association problem can be solved as a minimum cost multi-commodity flow problem. For each new commodity flow through the graph, a new association hypothesis is formed. Recall that edges were created from searching track fragment $o_{i}$ to neighboring track fragment $o_{j}$ and the cost of traversing one of these edges would be the cost of associating these two track fragments into one track fragment. In Pirsiavash et al. (2011), it was shown that the cost of the optimal solution in this directed acyclic graph (DAG) is convex and can be solved by solving $K+1$ shortest-path problems, where $K$ is the optimal number of track hypotheses. The algorithm begins by setting the initial flow $f$ to zero and then iterate through two steps:

1. Find the minimum-cost hypothesis path $\tau$ from $S$ to $T$ in $G(f)$
2. If total cost of the path is negative, update $f$ by pushing unit-flow along path $\tau$.

This is repeated until no negative cost path can be found. Each iteration increases the flow through the graph by 1 and decreases the cost of flow by the cost of hypothesis path $\tau$. For a graph with an optimal number of track fragment association hypotheses of $K$, the algorithm terminates after $K+1$ iterations having found a minimum cost flow, where any further flow from $S$ to $T$ would only server to increase the cost of flow through the network Pirsiavash et al. (2011).

Once the algorithm terminates, the track fragments that are part of the same hypothesis path through the graph are linked by taking the last detection in track fragment $o_{i}$ and linking it to the first detection in track fragment $o_{j}$. This linking is done in the same fashion as was done in the initial track fragment generation algorithm Algorithm 1. This process produces duplicate results in the same fashion as those returned from Algorithm 11, where there are two track fragment pointers pointing to the same linked list, like that depicted in Fig. 4. The duplicate track fragment pointers are pruned down to only include one per linked list, as was described and shown in Fig. 5, and the result set is returned as the set of current track hypotheses.

\section{Tracking Implementation}

As has been mentioned in earlier sections, multiplehypothesis tracking suffers from the combinatorial growth of its hypothesis space. To help mitigate this problem, a similar approach to that used in Hofmann et al. (2013) was adopted, where a hierarchy of stages in the tracking algorithm iteratively grows track fragments as they are passed from one stage to the next. Our approach utilizes track graphs as opposed to the use of the Hungarian algorithm for data association used in Hofmann et al. (2013). Our choice of track graphs was based upon how our problem differs from that in Hofmann et al. (2013). In our problem objects can appear or disappear at any position in both space and time. Whereas, Hofmann et al. (2013) only allows objects to enter and exit the tracking area along the edges of the image. In addition, our approach constructs track fragments in parallel, which, to our knowledge, has not been discussed previously.

This iteratively growing framework, shown in Algorithm 2, allows for the use of simple models on a majority of the object detection reports, and more complex models where greater ambiguity is present in the association hypotheses. For example, the first stage forms track fragments by linking object detection reports that are highly likely to belong to the same track. The determination of being highly likely to belong to the same track was accomplished by use of the differential rotation equation of the sun, as was used in Caballero and Aranda (2014) and developed in Howard et al. (1990), as a way to predict the location of a given detection at a later time. A depiction 


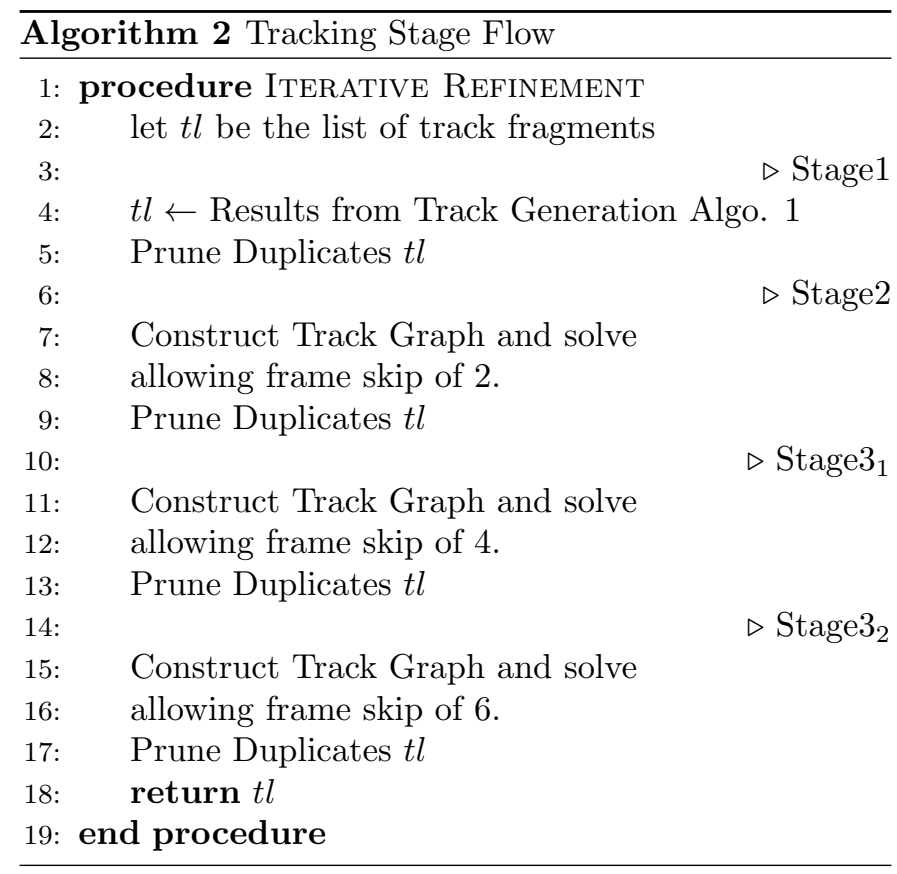

of the search area projected forward for a Coronal Hole was shown in Fig. 3 as the blue quadrilateral.

The steps used for the track fragment generation are shown in Algo. 1. This grouping of object detections into track fragments, significantly reduced the number of track fragments that the next stage needs to consider in its maximum a posteriori formulation through track graph creation and solving. As was shown in Kempton et al. (2014), this leads to a significant speedup over feeding every detection into the second stage as a track fragment. This is both due to the fact that the first stage processes object detection reports in parallel, and that the heuristic approach utilized in Algorithm 1 is significantly less computationally expensive when compared to the optimization of the global hypothesis, as is done in the second and third stages.

The second stage, as shown in Algorithm2, is given the list of track fragments produced and pruned in the first stage. These track fragments are associated into longer tracks by formulating the tracking task as a maximum a posteriori problem, which is solved using a track graph approach as discussed in Section 3. To construct the track graph, various parameters are considered that were not in the first stage of Algorithm 2. For example, if the track fragment is sufficiently long, meaning more than two object detections in length, the previous motion of vector is used to predict the next track fragment location, as opposed to the differential rotation used in Stage 1. In addition, the second stage allows the search for candidate track fragments to consider those that occur more than one time period in the future. This frame skip allowance, is an attempt to allow for missed detections; this is modeled in the frame skip model detailed later in Section 4.3.2. The details of the transition probabilities are discussed in more detail in Section 4.3 .
The third and fourth stages, as shown in Algorithm 2 , are similar to the second stage, in that each takes the list of pruned track fragments as input from the previous stage. However, a larger number of skipped frames are allowed in the third, and even longer in the fourth. In addition, these two stages also use the similarity of motion to compare the candidate track fragments with the search fragment, if they are sufficiently long enough, otherwise they only have the longer frame skip allowance when compared to Stage 2 .

The flow of the algorithm is such that in the fist stage of Algorithm 2, candidate detections were required to be immediate temporal neighbors of the searching detection. In the second stage of Algorithm 2, this was changed to allow candidates to be separated by up to two skipped time period frames. This means that the algorithm searches for track fragments that start up to two time frames after the end of the one being processed. The results from this second stage are again used as input into the next stage of Algorithm 2, where the frame gap is allowed to be up to four skipped frames. In the final stage of Algorithm 2, the limit on the number of skipped frames is extended to six.

This slowly growing allowance in the number of skipped frames gives preferential treatment to those track fragments that are close both spatially and temporally. Where only those track fragments that were not able to find a neighboring track fragment in the previous step are allowed to search across a greater gap of skipped frames. This process has the added benefit of limiting the number of track hypotheses that need to be tested at any one step in the algorithm, and thereby also reducing the computation time at that step.

\subsection{Markov Chain Parameters}

In Eq. 1 the posterior probability of the global hypothesis was rewritten as the product of the likelihood of an observation given the hypothesis, and the prior probability of the hypothesis. The likelihood function was then modeled as a Poisson probability distribution dependent up the change in the number of detections in a frame compared to the average change in the previous $n$ frame window. The likelihood function was then used in the calculation of the cost of observation in Eq. 7d. However, the prior probability of the hypothesis was shown to be a Markov chain in Eq3. with an initialization probability $P_{\text {entr }}$, used in Eq. $7 \mathrm{a}$ as the cost of a hypothesis entering the track graph at a specific track fragment. Similarly, a termination probability $P_{\text {exit }}$, is used in Eq. $7 \mathrm{~b}$ as the cost of a hypothesis exiting the track graph at a specific track fragment. Finally, a set of transition probabilities $P_{l i n k}\left(o_{k_{i+1}}, o_{k_{i}}\right)$ for each track fragment in the hypothesis, where each is used in Eq. $7 \mathrm{c}$ as the cost of a track fragment being in the hypothesis, is also part of the Markov chain.

In following sections we define the calculation of these previously mentioned parameters. In Section 4.2, the initialization and termination probability functions $\left(P_{\text {entr }}\right.$, 
$\left.P_{\text {exit }}\right)$ are described. In Section 4.3 the transition probability $P_{\text {link }}\left(o_{k_{i+1}}, o_{k_{i}}\right)$ is described, where its calculation differs depending upon which stage of the algorithm is being processed.

\subsection{Enter and Exit Probabilities}

In the previous work done in Hofmann et al. (2013), it was assumed that tracks can only start and end at the edges of the viewing area, with the exception of the starting and ending frames. This, however, does not hold true with the solar events in this work, as an event can start or end at almost any area of the solar surface in the viewing area shown in Fig. 7. As such, the enter and exit probabilities must be determined based upon the location and size of the event detections of interest. The heat maps in Figs. $7 \mathrm{a}$ and $7 \mathrm{~b}$ of the location of trajectories starting and ending are used in this calculation. These maps were initially produced using the results from the initial track fragment forming algorithm Algorithm 1, but have been refined with the results from the full implementation of our tracking work, as the algorithm's accuracy has improved.

In order to calculate the probability of a trajectory starting or ending at any given pixel location, we use the following calculation. Let $G=$ global maximum probability for either enter or exit, depending upon whether the enter or exit probability $\left(P_{\text {entr }}, P_{\text {exit }}\right)$ is being calculated. Then let $L=$ local minimum probability, or the pixel with the lowest probability inside the detection of interest. However, due to the fact that our heat map utilizes discrete pixels taken from a finite set of detections, there are areas in the constructed heat maps that contain a zero value when the probability of detection in those areas are greater than zero. In the cases that a detection's pixel intersects one of these areas, the average of all pixels in the detection of interest is assigned to $L$ instead of the local minimum. The calculation of the enter $P_{\text {entr }}\left(o_{i}\right)$ probability is the ratio of $L$ and $G$ :

$$
P_{\text {entr }}\left(o_{i}\right)=\frac{L}{G}
$$

Similarly for $P_{\text {exit }}\left(o_{i}\right)$, though the exit heat map is used as opposed to the enter heat map used in $P_{\text {entr }}\left(o_{i}\right)$. Also note, that when calculating $P_{\text {entr }}\left(o_{i}\right)$ of track fragment $o_{i}$, the first detection of the track fragment is used. Conversely, when calculating $P_{\text {exit }}\left(o_{i}\right)$ of track fragment $o_{i}$, the last detection in the track fragment is used. In either case, the probability of entering or exiting is always less than or equal to one.

\subsection{Transition Probabilities}

The transition probabilities $P_{l i n k}\left(o_{k_{i+1}}, o_{k_{i}}\right)$ are derived similarly to the work done in Hofmann et al. (2013), where they are comparing the similarity of the linked detection responses. There are three independent aspects of similarity: (1) appearance, (2) frame skip, and
(3) motion similarity. These three aspects of similarity are discussed in Sections 4.3.1, 4.3.2 and 4.3.3 respectively.

The transition probability is formulated differently depending upon the stage in question. There are two variants of this formulation:

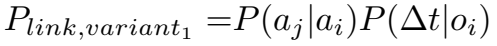

$$
\begin{aligned}
& P_{\text {link }_{\text {,variant }}}=P\left(a_{j} \mid a_{i}\right) P\left(\Delta t \mid o_{i}\right) P\left(v_{j} \mid v_{i}\right)
\end{aligned}
$$

Stage two from Algorithm 2 uses the first variant in Eq. 9a) stages three and four from Algorithm 2 use the second variant in Eq. $9 \mathrm{~b}$. In these variants, $P\left(a_{j} \mid a_{i}\right)$ is the appearance model, $P\left(\Delta t \mid o_{i}\right)$ the frame skip model, and $P\left(v_{j} \mid v_{i}\right)$ is the motion similarity model.

\subsubsection{Appearance Model}

The appearance model utilizes the image pixels that intersect the minimum bounding rectangle of either the first or last detection in a track fragment. To form the histogram $a_{j}$ of the candidate track fragment for linking with the search fragment, the first detection from track fragment $o_{j}$ is used. Recall that a search area is produced for candidate track fragments that are spatiotemporal neighbors of the last detection of the search track fragment $o_{i}$, similar to that described for Algorithm 1. A similar histogram creation process to that used in the creation of the histogram $a_{j}$ of the candidate track fragments is used to create the histogram $a_{i}$ of the search track fragment $o_{i}$, by taking the last detection in that fragment.

When spatiotemporal neighbors are compared, various image parameters are used to create the histograms of the pixels intersecting the minimum bounding rectangles. The image parameters that are used, were originally evaluated for use in solar Content-Based Image Retrieval of full disk images in Banda and Angryk (2010) and McInerney et al. (2013), and were later show to be useful in identifying similar regions of SDO AIA images in Banda et al. (2013). These pre-calculated parameters were later made available in Schuh et al. (2013) which was later extended to encompass a larger date range in Schuh and Angryk (2014). The labels used in this text, names, and equations can be seen in Table 1

The image parameters are calculated by dividing each 4096 by 4096 pixel SDO AIA image into 64 by 64 pixel cells and applying each of the formulas in Table 1 to the resultant area. The calculated value for each of the cells is the data which we use to construct histograms. This process effectively reduces the full resolution images to 10 sets of $64 x 64$ cell raster images for each wavelength image coming from the SDO AIA instrument. There are 9 wavelengths that we utilize, $94 \AA, 131 \AA, 171 \AA, 193 \AA, 211 \AA, 304 \AA, 335 \AA$, $1600 \AA$, and $1700 \AA$. In total, this gives us 90 different possible choices of image parameter and wavelength to use for our histogram creation. The specific parameters and wavelengths of images used for each event type processed will be discussed in Section 5.1 . 


\section{Track Beginning and Ending Heat Maps}

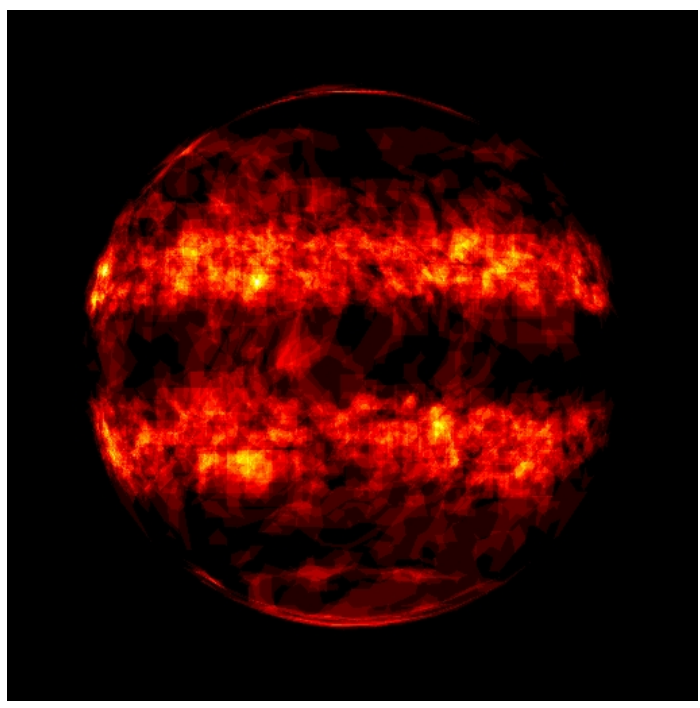

(a) Starting

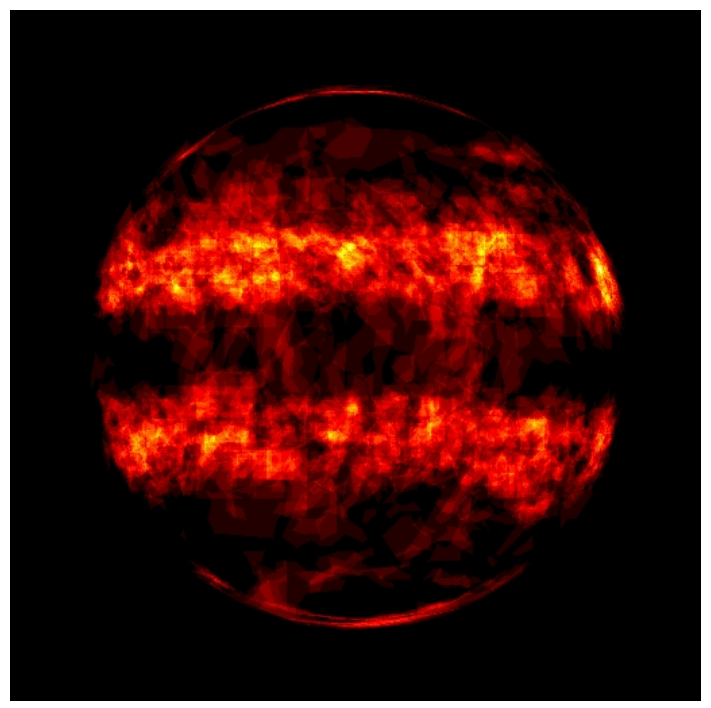

(b) Ending

Figure 7: Heat maps of where tracks start and end.

\section{Image Parameters}

\begin{tabular}{|c|c|c|}
\hline Label & Name & Equation \\
\hline P1 & Entropy & $E=-\sum_{i=0}^{L-1} p\left(z_{i}\right) \log _{2} p\left(z_{i}\right)$ \\
\hline P2 & Mean & $m=\frac{1}{L} \sum_{i=0}^{L-1} z_{i}$ \\
\hline P3 & $\begin{array}{l}\text { Std. Devia- } \\
\text { tion }\end{array}$ & $\sigma=\sqrt{\frac{1}{L} \sum_{i=0}^{L-1}\left(z_{i}-m\right)^{2}}$ \\
\hline $\mathbf{P} 4$ & Fractal Dim. & $D_{0}=-\lim _{\varepsilon \rightarrow 0}\left(\frac{\log N(\varepsilon)}{\log (\varepsilon)}\right)$ \\
\hline P5 & Skewness & $\mu_{3}=\sum_{i=0}^{L-1}\left(z_{i}-m\right)^{3} p\left(z_{i}\right)$ \\
\hline P6 & Kurtosis & $\mu_{4}=\sum_{i=0}^{L-1}\left(z_{i}-m\right)^{4} p\left(z_{i}\right)$ \\
\hline P7 & Uniformity & $U=\sum^{L-1} p^{2}\left(z_{i}\right)$ \\
\hline P8 & $\begin{array}{l}\text { Rel. } \\
\text { Smooth- } \\
\text { ness }\end{array}$ & $R=1-\frac{1}{1+\sigma_{2}(z)}$ \\
\hline P9 & T. Contrast & *sec Tamura et al. (1978) \\
\hline P10 & $\begin{array}{l}\text { T. Direction- } \\
\text { ality }\end{array}$ & *see Tamura et al. (1978) \\
\hline
\end{tabular}

Table 1: Image parameters, where $L$ stands for the number of pixels in the cell, $z_{i}$ is the intensity value of the $i$-th pixel, $m$ is the mean, and $p\left(z_{i}\right)$ is the histogram value of intensity value $z_{i}$. The fractal dimension is calculated on the box-counting method where $N(\varepsilon)$ is the number of boxes of side length $\varepsilon$ required to cover the image cell.
A subset of the available parameters were chosen because of their creating the greatest separation of the distribution of Hellinger distances calculated on the pixel histograms $a_{i}$ and $a_{j}$ using the equation:

$$
d\left(H_{1}, H_{2}\right)=\sqrt{1-\frac{1}{\sqrt{\bar{H}_{1} \bar{H}_{2} N^{2}}} \sum_{I}^{N} \sqrt{H_{1}(I) * H_{2}(I)}}
$$

Where

$$
\bar{H}_{k}=\frac{1}{N} \sum_{J}^{N} H_{k}(I)
$$

and $N$ is the total number of histogram bins $(N=20$ in all of our cases). Also, $H_{k}(I)$ is the value of the histogram bin at index $I$ of histogram $k$.

Two distributions distances were computed by comparing the image parameter histograms of detections belonging to the same track fragment and the histograms of detections belonging to different track fragments and are labeled as $A_{s}, \sigma_{s}$ for the same distribution and $A_{d}, \sigma_{d}$ for the different. With these distributions of distances assumed to be normal distributions, a model was produced to determine the visual similarity of two track fragments. The normal cumulative density function was used to find the probability of a given Hellinger distance being within 1 standard deviation of the reported value, when comparing two histograms $a_{j}$ and $a_{i}$. The probability was calculated for both of the distributions, same $N\left(x ; A_{s}, \sigma_{s}^{2}\right)$ and different $N\left(x ; A_{d}, \sigma_{d}^{2}\right)$ in the calculation of $P\left(a_{j} \mid a_{i}\right)$ :

$$
P\left(a_{j} \mid a_{i}\right)=\frac{N\left(A_{i j} ; A_{s}, \sigma_{s}^{2}\right)}{N\left(A_{i j} ; A_{s}, \sigma_{s}^{2}\right)+N\left(A_{i j} ; A_{d}, \sigma_{d}^{2}\right)}
$$


Note that $A_{i j}$ in Eq. 12 is the Hellinger distance of the histograms $a_{j}$ and $a_{i}$ of the detections of interest belonging to track fragments $o_{j}$ and $o_{i}$, similar to the model in Hofmann et al. (2013).

\subsubsection{Frame Skip Model}

The frame skip model $P\left(\Delta t \mid o_{i}\right)$, models the skipping of frames where detections are not reported in the dataset, but the tracked object still exists. This situation arises because no object detection algorithm is correct in identifying objects in an image a $100 \%$ of the time. This leads to the need to allow for missed detections of an object and to continue tracking an object across these gaps in detection.

In the model the $\Delta t$ is the gap between the last detection of the searching track fragment and the first detection in the candidate spaitiotemporal neighbor. It should be noted, that each detection is considered to have a period of time over which it is valid and another detection is not expected to be reported to the dataset. A detection with a start time is considered as having a skipped frame for each integer multiple of this period beyond the original time period considered as valid. This period is different for each event type in the dataset and is listed in Table 2 in Section 4.4. The model is then defined as an exponential model:

$$
P\left(\Delta t \mid o_{i}\right)=\prod_{t \in F}\left(1-P_{e x i t}\left(o_{i}\right)\right)
$$

In this, $F$ is the set of skipped frames in $\Delta t$. As was previously stated, one time period is allowed from the start of the last object detection report of the search track fragment $o_{i}$ and is considered the valid period of that detection. An additional period is added to the original period to search for detections that do not have a skipped frame, then another is added, and the search is repeated with the results considered as having one skipped frame. This process is repeated up to the maximum of $n$ skipped frames, where $n$ is dependent upon the limit set by the stage of Algorithm 2,

\subsubsection{Motion Model}

In the second variant of the transition probability calculation, Eq. 9b there is one more term, which considers the motion similarity of the two compared track fragments. This motion model, similar to Hofmann et al. (2013), the normalized movement vectors of the candidate track fragment $o_{j}$ and the search track fragment $o_{i}$ are calculated. This value is the normalized mean of the frame wise movement of the detections within the track fragments; these means are labeled as $v_{j}$ for the candidate track fragment and $v_{i}$ for the search track fragment. The model value is then calculated as:

$$
P\left(v_{j} \mid v_{i}\right)=1-\frac{1}{2}\left\|v_{j}-v_{i}\right\|
$$

This comparison of the Euclidean norm of movement differences, on the two track fragments, assumes that the motion of the detected objects are not changing direction abruptly. The result is the probability of the track fragments being the same object given their movement similarity.

\subsection{Indexing Detections by Time Domain Partitioning}

In the calculation of the cost of observation in Eq. $7 \mathrm{~d}$, which uses the likelihood of observation function described in Section 2.1.1, there is a requirement to rapidly find the average number of detections observed in discrete time frames. This necessitates the construction of an index structure that stores the detections seen, at a specified interval of time. Our solution, is to partition the temporal domain and store in the index the detections that intersect each partition. If no such structure was constructed, the best that could be hoped for is a list of all detections sorted on their time of occurrence.

In the case of a sorted list, this list would need to be searched for the first detection occurring at the time interval of interest, and traverse the list counting to the last. This scenario is not ideal, as a linear search would, on average, assuming the values of the list are equally likely to be searched for, require visiting $\frac{n}{2}$ elements. There does exist other searching algorithms for sorted lists, such as Binary search, which have time complexities of $O(\log N)$, though better options can be implemented to take advantage of the information we wish to retrieve from this indexed information. One such implementation would be to use a hash table, which would give a time complexity of $O(1)$ Cormen et al. (2001).

A major simplifying factor of the information that is intended to be retrieved from the index, is that the algorithm does not need to uniquely identify a specific detection. The algorithm is only concerned with the number of detections in a particular time interval, and how those intervals are distributed over the time domain is know at the time of index creation. Thus, there is no need to redistribute object detection reports among buckets, once one has a large number of detections placed into it, as access time to an individual detection in a bucket does not need amortized. This allows the insertion algorithm for the index to neglect to consider the number of detections in a bucket when inserting another detection into the index, as searching the bucket is not intended to take place.

In the implementation of the temporal index, the span of a frame is defined as a constant value for the event type being indexed. This span is a known value that is defined by the user. In our implementation, the average time between detections reported to the HEK for a given event type are used as this constant. This span value is used in the calculation of which bucket a specific detection belongs, and the spans are listed in Table 2 .

At the insertion of an object detection report, a global period variable is checked to see if the new detection falls inside the global period of the index. If the detection falls outside of the global period of the index, either partially or completely, the number of frames (buckets) beyond the 


\begin{tabular}{ll}
\hline Event Type & Time span (Seconds) \\
\hline Active Region & 12600 \\
Coronal Hole & 12600 \\
Emerging Flux & 9600 \\
Filament & 57600 \\
Sigmoid & 5300 \\
Sun Spot & 21000 \\
\hline
\end{tabular}

Table 2: The time span used as the valid time for each event detection.

current global period, that the detection falls, is calculated. The needed number of buckets are added to the index to accommodate the new detection. These new buckets are added at either the beginning or end of the index, depending upon whether the global time span of the index is to be expanded to a previous time or a later time. Once the buckets are added to the index, the global period is updated to reflect the new span that the index is valid for, either by moving the starting or ending time of the global period.

With the global period updated to be valid over the periods that the object detection report to be inserted is valid, the buckets that the detection report should be inserted are calculated. This is accomplished by first finding the starting frame by calculating the offset of the object detection report from the beginning of the global period and calculating how may periods that corresponds to. The detection pointer is then added to $n$ of the trailing buckets, where $n$ is the calculated frame span periods that the detection is valid. Once all object detection reports in the set of object detection reports $O$ are inserted, the buckets of the index represent a discrete time frame of detection. These time frames are then used in the calculation of the average number of detections per period of interest, used in the likelihood of observation. The likelihood is then used in the observation cost calculation in $7 \mathrm{~d}$. The calculation of which is now only requires $O(1)$ access to the first bucket of interest and $m$ bucket size reads for the entire calculation, where $m$ is the number of frames we wish to use in the averaging.

\subsection{Indexing Detections by Spatial Grid}

In the track fragment formation done in Algorithm 1 , object detection reports are grouped into track fragments. However, not all detections need to be considered as potential candidates for grouping with a particular detection report. A simple sort of the list of detection reports by start time could be applied and subsequent searches for candidates based upon time could be done. However, as discussed in Section 4.4, searching a time sorted list is a potentially expensive process, with a search being an $O(\log N)$ operation. When coupled with the cost of also testing the temporal candidates for spatial intersection with the search area, this approach becomes an unsatisfactorily expensive operation.
In practice, the spatial intersection test tends to be a very computationally expensive operation, so we attempt to minimize the need for this operation. To accomplish this, a fixed grid index was chosen as a spatiotemporal index for object detection reports. In this fixed grid index, each bucket of the grid is a sorted list of detection pointers of object detection reports, that intersect the particular grid location. The detection reports in each bucket are sorted by start time, making finding the first detections that temporally intersects the interval of interest an $\log m$ operation, where $m$ is the number of detection reports in the bucket. In practice, $m$ is significantly smaller than $N$, as detection reports are spread over the grid and do not all occur in the same position.

This fixed grid design was chosen because it only requires the spatial intersection of object detection reports to be calculated when building the index. This makes querying much less expensive, as the intersection of gird locations will only be calculated on the query quadrilateral shown in Fig. 3. instead of both the search quadrilateral and temporal candidate reports. There are also efficient indexing structures, such as $\mathrm{R}^{*}$ trees, that are good general solutions for indexing spatiotemporal objects, such as these detection reports, which can reduce the number of candidates that would have to be tested for spatial intersection. However, candidates after filtering still require a final refinement step of calculating the exact spatial intersection.

In addition to reducing the number of times an intersection is calculated on the detection reports, through the use of the fixed grid, we also constructed the grid in such a way that reduces both the number of buckets and the number of grid points that need to be tested for query quadrilateral and detection intersection. This is accomplished by scaling down the detection pixel space by a factor of 32. So, instead of a pixel space of 4096 by 4096 , the index uses a grid space of 128 by 128 . Since we are interested in detections in an approximate neighborhood, the inexact spatial representations of the detections that result from this scaling are acceptable side affects, for the reduction in computational cost. If a more accurate spatial representation of the detection reports were desired, the scaling factor could be reduced.

\subsection{Indexing Tracks by Spatial Grid}

Similar to the track fragment formation in Algorithm 1. the pairing of track fragments in remaining stages of Algorithm 2 require the ability to efficiently find spatialtemporally neighboring track fragments. To do this, a similar structure as the fixed grid structure that was used for individual detection reports in Section 4.5. was adapted for use with track fragments. The main difference between the two fixed grid approaches is the objects on which they operate. In the track fragment generation step that the report index was used, the fundamental object being processed was the object detection report. Where, in the remaining stages of Algorithm 2, the fundamental object 
being processed is the track fragment, which may contain multiple object detection reports.

In order to accommodate the differing structure of the data, the index of track fragments utilizes two of the 128 by 128 grids that the detection report index utilized. These two grids were used to store pointers of track fragments, instead of detection reports. One gird is constructed based upon the first detection report in the track fragment, whereas the other was based upon the last detection report in the track fragment. This is because we are only interested in where these track fragments are located at their beginning and ending points, when querying for spatiotemporal neighbors. Other than the storage of track fragment pointers, each of these two grids work the same as the detection report grid previously used.

\section{Experimental Results}

Our tracking approach was applied to solar data retrieved from the HEK in the range of January 2012 to December 2013. The following event types were retrieved:

- Active Region

- Coronal Hole

- Filament

- Sigmoid

- Sunspot

- Emerging Flux

However, since not all event types contain labels of track participation in the HEK, only a subset of these types were extensively evaluated for accuracy of our tracking approach. The event types that were evaluated are Active Regions and Coronal Holes, as these two types have tracking data present in the HEK dataset, which was produced by the Spatial Possibilist Clustering Algorithm (SPoCA) Verbeeck, C. et al. (2014).

\subsection{Experimental Details}

In our previous work Kempton et al. (2014), it was shown that increased speed and accuracy was gained by utilizing an algorithm that iteratively grows track fragments, when compared to a process that tries to solve all track hypotheses in one step. As is shown in Algorithm 2. our iterative approach first groups detections into track fragments with a simple heuristic in Algorithm 1. This step is then followed by steps that take track fragments and stitch them together into larger track fragments, by finding the optimal flow through a cost flow network described in Section 3 .

Also in our previous work, a single set of distributions were used for the appearance model, meaning that the two distributions $A_{s}, \sigma_{s}$ and $A_{d}, \sigma_{d}$ of Hellinger distances described in Section 4.3.1, were the same for all event

\begin{tabular}{|l|l|}
\hline Name & Definition \\
\hline MOTA & $\begin{array}{l}\text { Multiple Object tracking Accu- } \\
\text { racy: } 1-[\text { The ratio of the sum } \\
\text { of all errors (misses, false pos- } \\
\text { itive, mismatches) and the to- } \\
\text { tal number of objects in each } \\
\text { frame }] \text { Larger is better. Range } \\
{[0 \%, 100 \%]}\end{array}$ \\
\hline MT\% & $\begin{array}{l}\text { Mostly Tracked: Percentage of } \\
\text { the ground truth trajectories } \\
\text { that are covered by one track } \\
\text { output for more than } 80 \% \text { of its } \\
\text { length. Larger is better. Range } \\
{[0 \%, 100 \%]}\end{array}$ \\
\hline
\end{tabular}

Table 4: Definitions of Tracking Evaluation Metrics (according to Li et al. (2009) and Bernardin and Stiefelhagen (2008))

types, even though different wavelengths were used as input. Similarly, all histograms constructed for comparison, were utilizing the same image parameters, entropy, $4^{\text {th }}$ Moment (kurtosis), and uniformity. These parameters were chosen because they displayed the best separation of the two distributions $A_{s}, \sigma_{s}$ and $A_{d}, \sigma_{d}$, over all event types. However, a better approach would be to use the image parameters and distributions $A_{s}, \sigma_{s}$ and $A_{d}, \sigma_{d}$ that show the best separation for each event type.

In order to find the best set of image parameters to use in histogram construction, all of the combinations of image parameters, from one parameter up to four, or 386 combinations per wavelength, were evaluated for each event type. The wavelengths that were tested are $94 \AA, 131 \AA$, $171 \AA, 193 \AA, 211 \AA, 304 \AA, 335 \AA, 1600 \AA$, and $1700 \AA$. The wavelengths and image parameter combinations that were found to produce the best separation of the two distributions $A_{s}, \sigma_{s}$ and $A_{d}, \sigma_{d}$, for each event type are listed in Table 3 .

\subsection{Evaluation Metrics}

The metrics used to evaluate the accuracy of the tracking algorithm, in this work, are listed in Table 4. The definition of Mostly Tracked (MT) is from Li et al. (2009), where mostly tracked is when the ground truth trajectory is covered by one tracking output trajectory from the tracking algorithm, for greater than eighty percent of its length. This, however, leaves some ambiguity as to how switches in the identity of tracked output trajectories should be handled, when they later switch back to the original ground truth identity. We have made the assumption, that when a ground truth trajectory is covered by the same tracking output trajectory for a total number of frames that equates to eighty percent of its entire length, then we consider it mostly tracked.

It is our belief that Multiple Object Trajectory Accuracy (MOTA), from Bernardin and Stiefelhagen (2008), 


\begin{tabular}{cccccc}
\hline Event type & Wavelength & Param 1 & Param 2 & Param 3 & Param 4 \\
\hline \hline Active Region & $335 \AA$ & Standard Deviation & Uniformity & T. Contrast & T. Directionality \\
Coronal Hole & $335 \AA$ & Mean & Standard Deviation & Kurtosis & T. Contrast \\
Emerging Flux & $335 \AA$ & Standard Deviation & Skewness & Kurtosis & NULL \\
Filament & $94 \AA$ & Mean & Uniformity & T. Contrast & NULL \\
Sigmoid & $94 \AA$ & Mean & Kurtosis & T. Contrast & NULL \\
Sun Spot & $1600 \AA$ & Mean & Standard Deviation & Skewness & T. Contrast \\
\hline
\end{tabular}

Table 3: Wavelength and image Parameters used in appearance model for each event type.

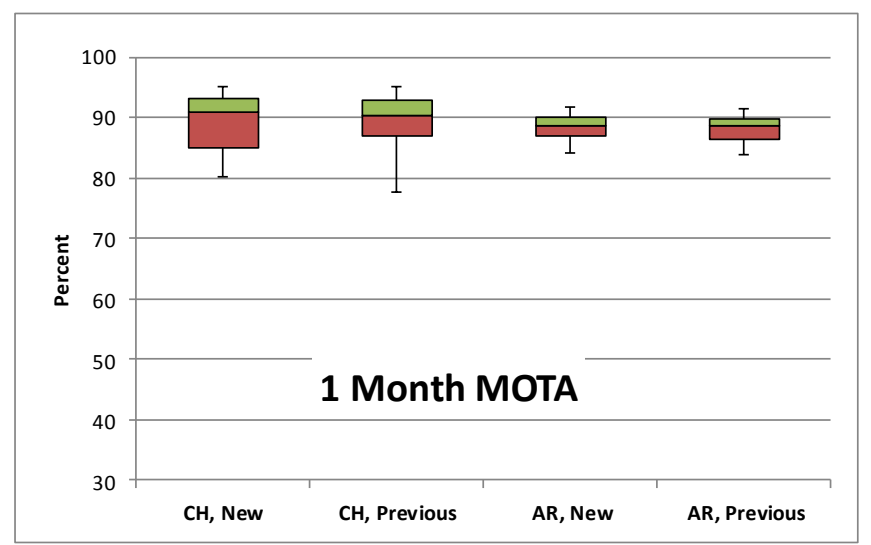

Figure 8: Distribution of Multiple Object Tracking Accuracy for 24 months of Coronal Hole and Active Region data.

provides a more accurate description of the accuracy of the tracking algorithm. The MOTA metric sums all the errors at each time step (misses, false positive, mismatches) and then computes the ratio of errors to the total number of objects over all time steps. This process gives the total error rate $E_{t o t}$, and $1-E_{\text {tot }}$ is the resulting MOTA metric value. This metric gives a clearer picture of what the accuracy of the algorithm is, since the error at each time step is calculated.

\subsection{Tracking Performance}

The two years of data that was used for this work was split into twenty-four one month subsets and the tracking algorithm was applied to each. Two versions of the algorithm were used, the previous version which used only one global distribution and image parameter set for the appearance model, as was done in Kempton et al. (2014), and one where each event type used its own distribution and image parameter set, which was found to be the best for them. The parameters used are listed in Table 3 . The output trajectories produced for each month were compared to the SPoCA labels, produced by Verbeeck, C. et al. (2014), present in the retrieved data from the HEK. The results for both algorithms are listed inf Figs. 8 and 9 . In the graphs the whiskers represent the $\min / \max$ of the data and the boxes are the second and third quartile, with the boundary between them being the median of the results. As can be seen in Figure 8, the current changes of us-

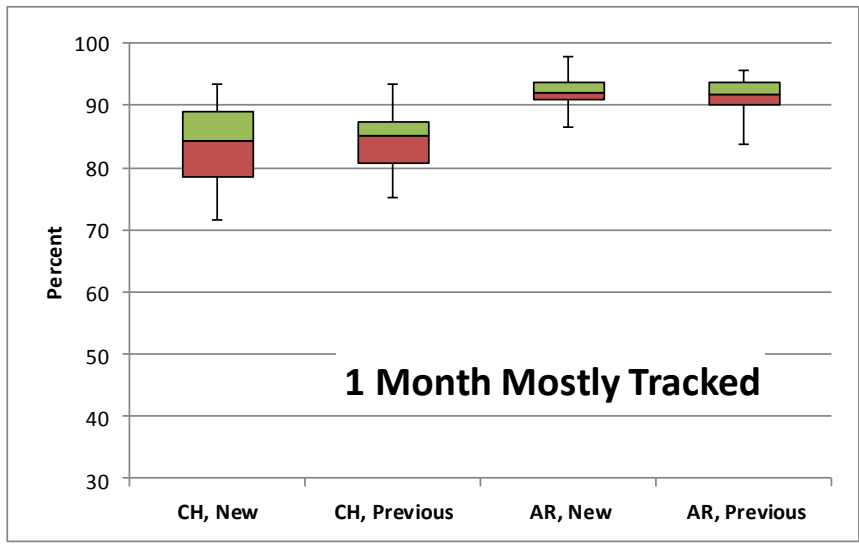

Figure 9: Distribution of the percentage of mostly tracked trajectories for 24 months of Coronal Hole and Active Region data.

ing different distributions, parameter sets, wavelengths for both Coronal Holes and Active Regions, produces an overall tighter bound on the total range of MOTA values seen across all months. There is an increase in the total range of 0.1 for MOTA values with Active Regions and a decrease in the total range of 2.6 for Coronal Holes being seen, which is an overall decrease. The maximum MOTA value is the same for both versions of the algorithm on Coronal Holes, at 95.1\%. However, we see a slight improvement in the maximum MOTA for active Regions, with the new at $91.8 \%$ versus $91.5 \%$ with the previous version.

In Figure 9, Active Regions see a 0.6 decrease in the range of values seen in the new implementation and an increase in the range of values seen of 3.7 for Coronal Holes. The maximum values seen for Mostly Tracked increases to $97.8 \%$ versus $95.7 \%$ with Active Regions. Like with MOTA, no improvement in the maximum value of Mostly Tracked is seen with Coronal Holes, with both versions reporting a $93.5 \%$ maximum.

\section{Conclusion}

In this work, we provided some of the implementation details that were left out of Kempton et al. (2014) for the sake of brevity, and introduced some changes in the way the likelihood function of observation was implemented. Additionally, we introduced the use of custom sets of both wavelength of images used and image parameters used for 
each event type. These custom sets were also accompanied with distributions of $A_{s}, \sigma_{s}$ and $A_{d}, \sigma_{d}$ exclusively calculated for each event type, where previous implementations used the same distributions for all event types. These improvements saw incremental improvements of the maximum accuracy measure values that were seen in our experimental results, in addition to slightly increased variance for some of the measures.

\section{Future Work}

Though the SPoCA labeled data is a good starting point for comparison, these labels are available for only two of the data types. As such, we have begun to gather human-labeled tracks for comparison in future work. It is our belief that using an ensemble of labels from multiple human labelers will produce adequate results for use in comparison testing with tracking algorithms, even with non-experts being utilized.

Additional work can, and should, be conducted in the exploration of using different histogram comparison methods for use in the appearance model discussed in Section 4.3.1. Similarly, more work should be conducted in exploring the use of multiple wavelengths and image parameters in the construction of the histograms used to compare the similarity of two track fragments. The current work does utilize different wavelengths for each event type and different image parameters from the same wavelength image, but more can be done in taking different combinations of parameters from multiple wavelength images.

It is our plan to utilize at least three additional comparison measures to the Hellinger measure utilized in this work. These histogram comparison measures include Correlation, Chi-Square, and Intersection. These different measures will be tested across multiple combinations of image parameters from differing wavelength images to find a robust combination for each event type.

It should also be noted, that the appearance model utilized in this work made the relatively naïve assumption that the distributions of histogram comparison values were normally distributed, when considering the comparison of track fragments that are the same tracked object and those that are different tracked objects. This assumption should be explored further to determine if this is indeed a valid assumption. To that end, there is the possibility that more complex models would be better suited for this classification task, and other models should be explored.

Finally, it is our intent to continue improving our algorithm and eventually releasing both our results and code. For now, we encourage anyone interested in the dataset produced by our algorithm, to contact the authors for the latest results.

\section{Acknowledgments}

This work was supported by National Aeronautics and Space Administration (NASA) grant award No.
NNX11AM13A, and by National Science Foundation (NSF) grant award No. 1443061.

\section{References}

Banda, J., Angryk, R., 2010. An experimental evaluation of popular image parameters for monochromatic solar image categorization, in: FLAIRS Confernence, 2010 Twenty-Third, pp. 380-385.

Banda, J., Liu, C., Angryk, R., 2013. Region-based querying of solar data using descriptor signatures, in: Data Mining Workshops (ICDMW), 2013 IEEE 13th International Conference on, pp. 1-7. doi 10.1109/ICDMW . 2013.127.

Bernardin, K., Stiefelhagen, R., 2008. Evaluating multiple object tracking performance: The clear mot metrics. J. Image Video Process. 2008, 1:1-1:10. URL: http://dx.doi.org/10.1155/2008/ 246309 doi $10.1155 / 2008 / 246309$.

Caballero, C., Aranda, M., 2014. Automatic tracking of active regions and detection of solar flares in solar euv images. Solar Physics 289, 1643-1661. URL: http://dx.doi.org/10.1007/ s11207-013-0415-4 doi 10.1007/s11207-013-0415-4

Chong, C.Y., 2012. Graph approaches for data association, in: Information Fusion (FUSION), 2012 15th International Conference on, pp. 1578-1585.

Chong, C.Y., Castanon, G., Cooprider, N., Mori, S., Ravichandran, R., Macior, R., 2009. Efficient multiple hypothesis tracking by track segment graph, in: Information Fusion, 2009. FUSION '09. 12th International Conference on, pp. 2177-2184.

Cormen, T.H., Stein, C., Rivest, R.L., Leiserson, C.E., 2001. Introduction to Algorithms. 2nd ed., McGraw-Hill Higher Education.

Hofmann, M., Haag, M., Rigoll, G., 2013. Unified hierarchical multiobject tracking using global data association, in: Performance Evaluation of Tracking and Surveillance (PETS), 2013 IEEE International Workshop on, pp. 22-28. doi 10.1109/PETS.2013. 6523791 .

Howard, R., Harvey, J., Forgach, S., 1990. Solar surface velocity fields determined from small magnetic features. Solar Physics 130, 295-311. URL: http://dx.doi.org/10.1007/BF00156795, doi 10 . 1007/BF00156795

Hurlburt, N., Cheung, M., Schrijver, C., Chang, L., Freeland, S., Green, S., Heck, C., Jaffey, A., Kobashi, A., Schiff, D., Serafin, J., Seguin, R., Slater, G., Somani, A., Timmons, R., 2012. Heliophysics event knowledgebase for the solar dynamics observatory (sdo) and beyond, in: Chamberlin, P., Pesnell, W., Thompson, B. (Eds.), The Solar Dynamics Observatory. Springer US, pp. 6778. URL: http://dx.doi.org/10.1007/978-1-4614-3673-7_5, doi 10.1007/978-1-4614-3673-7\_5

Kempton, D., Pillai, K., Angryk, R., 2014. Iterative refinement of multiple targets tracking of solar events, in: Big Data (Big Data), 2014 IEEE International Conference on, pp. 36-44. doi 10.1109/ BigData.2014.7004402

Li, Y., Huang, C., Nevatia, R., 2009. Learning to associate: Hybridboosted multi-target tracker for crowded scene, in: Computer Vision and Pattern Recognition, 2009. CVPR 2009. IEEE Conference on, pp. 2953-2960. doi 10.1109/CVPR.2009.5206735

Martens, P., Attrill, G., Davey, A., Engell, A., Farid, S., Grigis, P., Kasper, J., Korreck, K., Saar, S., Savcheva, A., Su, Y., Testa, P., Wills-Davey, M., Bernasconi, P., Raouafi, N.E., Delouille, V., Hochedez, J., Cirtain, J., DeForest, C., Angryk, R., De Moortel, I., Wiegelmann, T., Georgoulis, M., McAteer, R., Timmons, R., 2012. Computer vision for the solar dynamics observatory (sdo). Solar Physics 275, 79-113. URL: http://dx.doi.org/10.1007/ s11207-010-9697-y doi 10.1007/s11207-010-9697-y

McInerney, P., Banda, J., Angryk, R., 2013. On using sift descriptors for image parameter evaluation, in: Data Mining Workshops (ICDMW), 2013 IEEE 13th International Conference on, pp. 3239. doi 10.1109 /ICDMW.2013.123

Meng, J., Raghunathan, A., Chakradhar, S., Byna, S., 2010. Exploiting the forgiving nature of applications for scalable parallel execution, in: Parallel Distributed Processing (IPDPS), 2010 IEEE International Symposium on, pp. 1-12. doi 10.1109/IPDPS. 2010. 5470469 . 
Pirsiavash, H., Ramanan, D., Fowlkes, C., 2011. Globally-optimal greedy algorithms for tracking a variable number of objects, in: Computer Vision and Pattern Recognition (CVPR), 2011 IEEE Conference on, pp. 1201-1208. doi 10.1109/CVPR.2011.5995604 Schuh, M., Angryk, R., 2014. Massive labeled solar image data benchmarks for automated feature recognition, in: Big Data (Big Data), 2014 IEEE International Conference on, pp. 53-60. doi 10.1109/BigData.2014.7004404.

Schuh, M., Angryk, R., Pillai, K., Banda, J., Martens, P., 2013. A large-scale solar image dataset with labeled event regions, in: Image Processing (ICIP), 2013 20th IEEE International Conference on, pp. 4349-4353. doi 10.1109/ICIP. 2013.6738896.

Tamura, H., Mori, S., Yamawaki, T., 1978. Textural features corresponding to visual perception. Systems, Man and Cybernetics, IEEE Transactions on 8, 460-473. doi 10.1109/TSMC.1978. 4309999

Verbeeck, C., Delouille, V., Mampaey, B., De Visscher, R., 2014. The spoca-suite: Software for extraction, characterization, and tracking of active regions and coronal holes on euv images. A\&A 561, A29. URL: http://dx.doi.org/10.1051/0004-6361/201321243 doi $10.1051 / 0004-6361 / 201321243$

Zhang, L., Li, Y., Nevatia, R., 2008. Global data association for multi-object tracking using network flows, in: Computer Vision and Pattern Recognition, 2008. CVPR 2008. IEEE Conference on, pp. 1-8. doi 10.1109/CVPR.2008.4587584 